\title{
Optimizing the Shape of Planetary Rover Wheels using the Discrete Element Method and Bayesian Optimization
}

\author{
Leon Stubbig* and Roy Lichtenheldt \\ German Aerospace Center (DLR) \\ Institute of System Dynamics and Control \\ Münchener Straße 20, 82234 Weßling, Germany \\ e-mail : leon.stubbig@dlr.de, web page : https://www.dlr.de/sr/
}

\begin{abstract}
The Martian Moons eXploration (MMX) mission will include a rover to conduct in situ science and exploration on the Martian moon Phobos [1]. The rover is being developed as a joint project by CNES and DLR. The rover's locomotion system and especially its wheels require special consideration as wheeled locomotion has never been employed in a comparably low gravity environment. Wheel design for Phobos is complicated by the little researched behavior of regolith under low gravity and the inability to conduct milli-g experiments of a sufficient duration on Earth, necessitating the assumption of a worst-case soft soil. This paper shows a novel, automated approach to design the shape of rover wheels. It uses high-fidelity simulation models based on the Discrete Element Method [2] and a Bayesian Optimization algorithm [3]. As the outer wheel diameter and wheel width are given by mission constraints, four parameters were selected to describe the wheel shape: grouser height, grouser number, grouser chevron angle and rim curvature. On Phobos, three main scenarios are important to ensure the rover's successful operation: Driving forward, backward and uphill of up to 10 degrees. The optimization loop generates wheels within wide limits of the four wheel shape parameters. Each wheel is simulated with free slip conditions in each scenario. A simulation on flat soil is shown in Figure 1. The performance of each wheel is assessed based on the travelled distance. The optimization is run for 115 iterations, which corresponds to about one month of wall-clock time. It yields an optimized wheel shape as well as a dataset showing the dependence of performance on the wheel shape parameters. In contrast to the prototype wheel, see Figure 2, the optimized wheel shape shown in Figure 3 has larger flat grousers. The inward curved rim shape was confirmed. The optimized wheel performs $64 \%$ better than the previous prototype. The optimized shape and parameter relations will now guide the design of the wheels for the MMX mission.
\end{abstract}

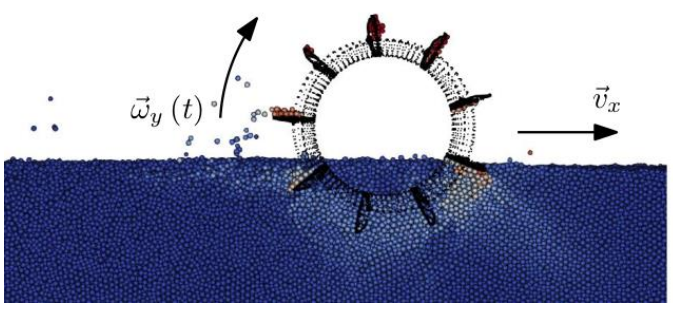

Figure 1: Wheel simulation on flat soil

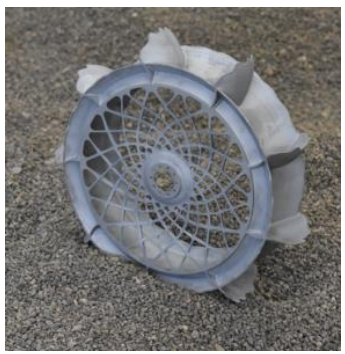

Figure 2: Prototype

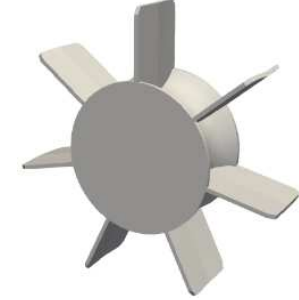

Figure 3: Optimized wheel shape

\section{REFERENCES}

[1] S. Ulamec, P. Michel, M. Grott et al., A Rover for the MMX Mission to Phobos, $70^{\text {th }}$ International Astronautical Congress (IAC), 2019.

[2] R. Lichtenheldt, S. Ono, L. Stubbig, Large Scale Discrete Element Simulation Campaigns Simulating Extraterrestrial Soils in partsival, $14^{\text {th }}$ World Congress on Computational Mechanics (WCCM), 2021.

[3] C. Williams, C. E. Rasmussen, Gaussian Processes for Machine Learning, MIT Press, 2006. 